\title{
DEVELOPMENT AND CHARACTERIZATION OF DOXORUBICIN AND SIRNA ENCAPSULATED CHITOSAN NANOPARTICLES
}

\author{
TAIHASEEN MOMIN ${ }^{1}$, ARVIND GULBAKE ${ }^{*}$ \\ ${ }^{1}$ Centre for Interdisciplinary Research, D. Y. Patil Education Society, Institution Deemed to be University, Kolhapur, Maharashtra, India \\ Email: arvind.gulbake@gmail.com
}

Received: 20 Apr 2020, Revised and Accepted: 30 Jul 2020

\begin{abstract}
Objective: Chitosan nanoparticles (ChNP's) have been widely studied for drug and gene delivery. In this study, we prepared ChNP's for co-delivery of doxorubicin (DOX) and siRNA for cancer treatment.

Methods: The ionic gelation method was used to develop ChNP's. The positively charged DOX and negatively charged siRNA encapsulated into ChNP's. The particle size and zeta potential of the developed ChNP's were studied by particle size analyzer and morphology was examined by TEM. Encapsulation of DOX in ChNP's was confirmed by FTIR spectroscopy. The encapsulation efficiency and in vitro release of DOX were studied by UVVis spectrophotometry. The siRNA loading into ChNP's was confirmed by gel retardation assay.

Results: The developed ChNP's showed particle size ranged from $127 \pm 6.5$ to $215 \pm 8.5 \mathrm{~nm}$ with zeta potential ranged from $16.5 \pm 0.3$ to $25.8 \pm 0.3$. Transmission Electron Micrograph showed DOX and siRNA encapsulated ChNP's are polydisperse and spherical in nature. FTIR study confirmed the binding of DOX with ChNP's with absorption peaks at $1016 \mathrm{~cm}^{-1}, 1316 \mathrm{~cm}^{-1}, 1412 \mathrm{~cm}^{-1}, 1645 \mathrm{~cm}^{-1}$ and $3370 \mathrm{~cm}^{-1}$. The TPP:Ch ratio $0.1: 0.5 \mathrm{showed}$ the highest encapsulation efficiency $69 \pm 3.24 \%$, with initial burst release and then sustained or slow release of DOX. Agarose gel retardation study confirmed the encapsulation of siRNA in ChNP's by retarded migration of siRNA-ChNP's in comparison with naked siRNA.
\end{abstract}

Conclusion: The developed ChNP's successfully encapsulated the DOX and siRNA and showed the sustain release of DOX. In conclusion, our study shown that ChNP's is having a potential of co-loading of DOX-siRNA as an efficient drug delivery system for the treatment of various cancers such as colorectal cancer, breast cancer etc.

Keywords: Multidrug Resistance (MDR), Chitosan, Doxorubicin, siRNA, Co-Delivery

(C) 2020 The Authors. Published by Innovare Academic Sciences Pvt Ltd. This is an open access article under the CC BY license (http://creativecommons.org/licenses/by/4.0/) DOI: http://dx.doi.org/10.22159/ijap.2020.v12s4.40105. Journal homepage: https://innovareacademics.in/journals/index.php/ijap

\section{INTRODUCTION}

Now a days, cancer is one of the main causes of death worldwide. Conventional therapies such as radiotherapy, surgery, chemotherapy and a combination of them are used to treat cancer [1]. Chemotherapy is one of the most commonly used treatment in which chemotherapeutics are used for treatment of cancer. Some most common chemotherapeutics such as 5-FU, paclitaxel, DOX are capable for suppression of tumor growth [2, 3].

DOX is an anthraquinone, hydrophilic anticancer drug that binds to topoisomerase, which induces apoptosis in different malignant cancer. However, problems related to acquired resistance and their acute cardiotoxicity, low penetration and limited distribution in solid tumor $[4,5]$. Due to this side effects use of free DOX in the biomedical field is still limited. An alternative approach is to reduce side effects encapsulate DOX within a positively charged nanocarrier. Nanocarrier based drug delivery systems offer several advantages, including the greater surface area of nanoparticles, due to reduced size, sustained drug release rate, improvement in biodistribution, and reduction in side effects [6, 7]. Recently, nanocarriers are used for drug delivery are polymeric nanoparticles, micelles, dendrimers etc. Polymeric nanoparticles are most studied carriers in drug delivery, which exhibits pH-responsive, drug retaining ability during circulation and releasing it at the tumor site. A major problem with chemotherapeutic drugs is the gradually acquired multidrug resistance (MDR) due to the overexpression of active drug efflux pumps, which reduces the therapeutic efficacy. Even the use of siRNAs that target particular genes has been shown to reduce resistance problems. However, delivering siRNA to tumor cells is a major challenge because serum nucleases degrade siRNA and are immediately cleared by the reticuloendothelial system (RES) [8, 9]. The Discovery of RNA interference (RNAi)-based gene silencing has drawn much attention to cancer therapy due to its unique properties to silence a broad range of genetic targets. RNA interference (RNAi) is a cellular mechanism for gene suppression induced by siRNA in which $21-23$ base pairs sequence of siRNA complementary to its target mRNA, which acts as a post-transcriptional regulator [10]. For clinical success, there is a major challenge associated with siRNA delivery at target site and cellular uptake. Some physiological and biological barriers are preventing their delivery at target site. But still, most practical applications need a carrier to transport the siRNA via systemic application to the target site [11-13].

Co-delivery of siRNA and encapsulated chemotherapeutic drugs is a promising approach to improve therapeutic efficacy. Various studies have shown that nanocarrier-based co-delivery of drugs with siRNA against the MDR genes suppressed P-glycoprotein (P-gp) expression which increases the retention of encapsulated drugs $[8,14]$.

$\mathrm{Ch}$ is a naturally occurring linear cationic polymer prepared through $\mathrm{N}$-deacetylation of chitin isolated from seaweeds. Recently, it has increasing attention in pharmaceutical and biomedical applications because of its biocompatibility, biodegradability, non-toxicity, cationic properties and bioadhesive characteristics. Thus, ChNP's are of great significance as drug delivery systems for cancer therapy [5, 6]. The ChNP's can be formulated through various techniques, such as coacervation, co-precipitation, solvent evaporation, ionic gelation, and microemulsion. In ionic gelation method, several cross-linking agents such as sodium tripolyphosphate and glutaraldehyde are used, which provide an efficient network to entrap the drug molecules into the nanoparticles [7, 15]. Here, we developed DOX and siRNA encapsulated ChNP's in order to create an efficient drug delivery system. Physicochemical properties of the DOX and siRNA encapsulated ChNP's were studied, such as particle size, surface charge, percentage encapsulation efficiency and in vitro-drug release, gel retardation assay.

\section{MATERIALS AND METHODS}

\section{Materials}

Low mol. wt. Ch (Mol. Wt. 50,000-190, 000 Da, Deacetylation Degree $75-85 \%)$, sodium tripolyphosphate (TPP) was purchased from 
Sigma-Aldrich (Mumbai, India), Acetic acid, Tween 80, $1 \mathrm{M} \mathrm{NaOH,}$ $\mathrm{HCl}$, Agarose gel electrophoresis kit was purchased from Himedia Laboratories Pvt. Ltd, Mumbai, India. DOX Hydrochloride was received as a gift sample from Sun Pharma Advance Research co Ltd, India. The siRNA was purchased from Santacruze Biotech, India. All other chemicals used were of analytical grade. Double distilled water (DDW) and RNase free water used throughout the study wherever needed.

\section{Preparation of ChNP's}

ChNP's were prepared via ionic gelation method established by Calvo et al. [16] Ch solutions $(0.3 \%, 0.4 \%, 0.5 \%, 0.6 \% \mathrm{w} / \mathrm{v}, \mathrm{pH}-5.5)$ were prepared by dissolving $\mathrm{Ch}$ in $1 \% \mathrm{v} / \mathrm{v}$ glacial acetic acid. TPP solution $(0.1 \% \mathrm{w} / \mathrm{v})$ was prepared by dissolving TPP in deionized water. ChNP's were prepared by adding TPP solution dropwise into Ch solutions at room temperature with constant magnetic stirring for $1 \mathrm{hr}$. Centrifugation was performed at $20,000 \mathrm{rpm}$ at $10^{\circ} \mathrm{C}$ for 30 min to collect nanoparticles. The supernatant was discarded and pellets of nanoparticles re-suspended in filtered deionized water.

\section{Preparation of DOX encapsulated ChNP's}

After optimization of Ch and TPP ratio 0.5:0.1 were selected for DOX encapsulation. To prepare DOX encapsulated ChNP's, DOX solution (Ch:DOX-10:1) were added into aqueous solution of TPP. Prepared TPP-DOX solution dropwise added into Ch solution under constant magnetic stirring for $1 \mathrm{hr}$. at R. T.

\section{Preparation of DOX and siRNA encapsulated ChNP's}

For preparation of DOX and siRNA encapsulated ChNP's, DOX (Ch: DOX-10:1) and $5 \mu \mathrm{l}$ of siRNAs $(20 \mu \mathrm{g} / \mu \mathrm{l})$ was added to $1.5 \mathrm{ml}$ aqueous solution of TPP $(0.1 \% \mathrm{w} / \mathrm{v})$, then this solution was dropwise added into $\mathrm{Ch}(0.5 \%, \mathrm{pH}-5.5)$ solution under magnetic stirring at R. T. Then particles were incubated at room temperature for another $30 \mathrm{~min}$ before further analysis. All solutions were made in RNase-free distilled water and autoclaved before use. To prevent photodegradation of DOX, glassware's was used either amber color or covered with aluminum foil.

\section{Characterization}

\section{Physicochemical characterization}

Nanoparticles diameter, polydispersity index and zeta potential were determined by Malvern Zetasizer Ver. 7.12 (Malvern Instruments Ltd, UK). The aqueous dispersions of the nanoparticles were measured at $25^{\circ} \mathrm{C}$ with a scattering angle of $90^{\circ}$.

\section{Morphology of nanoparticles}

The ChNP's morphology was examined by transmission electron microscopy at $200 \mathrm{kv}$ (JEOL Model JEM-2100).

\section{Fourier transform infrared spectroscopy (FTIR)}

Fourier transform infrared (FTIR) spectra were analyzed using an Alpha Bruker, Germany in the range $4000-400 \mathrm{~cm}^{-1}$. The DOX, ChNP's, DOX-ChNP's samples were mixed with $\mathrm{KBr}$ and form a thin pellet to investigate the functional group.

\section{Encapsulation efficiency of DOX}

The encapsulation efficiency of DOX into ChNP's was measured using a UV-vis spectrophotometer at $480 \mathrm{~nm}$. The free DOX in supernatant recovered from centrifugation $\left(20,000\right.$ at $10{ }^{\circ} \mathrm{C}$ for 30 min) were quantified by measuring its absorbance at $480 \mathrm{~nm}$ wavelength with a dual beam Agilent Technologies Cary 60 UV-Vis spectrophotometer.

$$
\text { Encapsulation efficiency }(\%)=\frac{\mathrm{C}(\text { sample })-\mathrm{C}(\text { supernatant })}{\mathrm{C}(\text { sample })} \times 100
$$

Where, C (sample) is the concentration of DOX added and C (supernatant) is the concentration of DOX in the supernatant (6).

\section{In vitro release study of DOX}

$4 \mathrm{ml}$ of DOX loaded ChNP's were dispersed in a freshly prepared phosphate-buffered saline (PBS; $\mathrm{pH}=7.4$ ) as a release medium in a dialysis membrane sac (molecular weight cut-off $12 \mathrm{kDa}$ ). The ChNP's containing sac dialysis in $50 \mathrm{ml}$ PBS (pH-7.4) as released media. The beaker was placed in a shaking incubator at $37^{\circ} \mathrm{C}$ under mild agitation. For each sample, $5 \mathrm{ml}$ of the release medium was withdrawn at prearranged time intervals and replaced with the same amount of medium every time. The amount of released DOX in the medium was assessed at absorbance $(\lambda=480 \mathrm{~nm})$ by UV-Vis spectrophotometry. The medium which was collected from plain NPs (without DOX) were used as the negative control. The percentage of cumulative DOX release was calculated according to the standard curve [10].

\section{Confirmation of siRNA encapsulation in ChNP's by Agarose gel retardation assay}

The binding of siRNA into ChNP's was confirmed by agarose gel retardation assay. Naked siRNA and prepared ChNP's, ChNP'ssiRNA samples were taken for loading. Before loading into the agarose gel, all samples were incubated and shaken gently at $37^{\circ} \mathrm{C}$ for $30 \mathrm{~min}$; after that adding $5 \mu \mathrm{l}$ of loading buffer into all samples. The samples were electrophoresed in $1 \%(\mathrm{w} / \mathrm{v})$ agarose gel containing ethidium bromide $(2 \mathrm{mg} / \mathrm{ml})$ at a constant voltage of $120 \mathrm{~V}$ for $20 \mathrm{~min}$. The results was observed under the UV transilluminator [17].

\section{RESULTS AND DISCUSSION}

ChNP's were prepared according to the ionic gelation method using negatively charged polyanion crosslinker TPP, which binds reversibly to positively charged $\mathrm{Ch}$ through electrostatic interaction at room temperature. This is an organic solvent-free method with a better ability to encapsulating hydrophilic drug DOX and siRNA $[5,15]$.

\section{Physicochemical characterization}

The DOX and siRNA encapsulated ChNP's were optimized on the basis of size distribution, surface charge, polydispersity index and encapsulation efficiency (table 1). The results revealed that Ch:TPP ratio 0.1:0.5 was found optimum on the basis of maximum percentage DOX encapsulation efficiency $(69 \pm 3.24 \%)$ with optimum size and distribution $(192 \pm 8.1$ and $0.62 \pm 0.05)$.

\section{Morphology of nanoparticles}

The morphology of ChNP's was confirmed by TEM in which ChNP's were seen to be spherical with solid dense structure. The nanoparticles were appeared to be considerably smaller than the average particle size observed with the particle size analyzer.

Table 1: Optimization of DOX and siRNA encapsulated ChNP's

\begin{tabular}{lllll}
\hline TPP: Ch (ratio) & Particle size (nm) & Zeta potential (mV) & PDI & Encapsulation efficiency \\
\hline $0.1: 0.3$ & $127 \pm 6.5$ & $16.5 \pm 0.3$ & $0.86 \pm 0.03$ & $39 \pm 2.14 \%$ \\
$0.1: 0.4$ & $158 \pm 6.7$ & $22.4 \pm 0.5$ & $0.63 \pm 0.05$ & $52 \pm 2.84 \%$ \\
$\mathbf{0 . 1 : 0 . 5}$ & $\mathbf{1 9 2 \pm 8 . 1}$ & $\mathbf{2 4 . 6 \pm 0 . 3}$ & $\mathbf{0 . 6 2 \pm 0 . 0 5}$ & $\mathbf{6 9 \pm 3 . 2 4 \%}$ \\
$0.1: 0.6$ & $215 \pm 8.5$ & $25.8 \pm 0.3$ & $0.96 \pm 0.05$ & $65 \pm 2.78 \%$ \\
\hline
\end{tabular}

Values represent mean $\pm S D(n=3)$ 


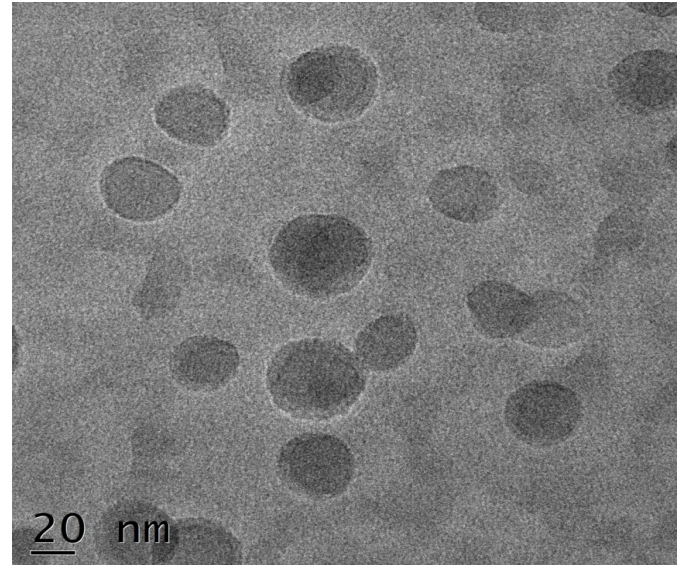

Fig. 1: Transmission electron microscopy of DOX and siRNA encapsulated ChNP's

\section{FTIR analysis}

FTIR spectroscopy is an important tool used to determine the functional groups in a molecule. In fig. 2 shown FTIR spectra of ChNP's, DOX, ChNP's-DOX. In ChNP's wider peak was observed at $3302 \mathrm{~cm}^{-1}$ due to the development of hydrogen bonding and shoulder peak at $1624 \mathrm{~cm}^{-1}$. In DOX-loaded ChNP's the absorption bands appeared at $1016 \mathrm{~cm}^{-1}$ and $1645 \mathrm{~cm}^{-1}$ are related to the DOX [18].

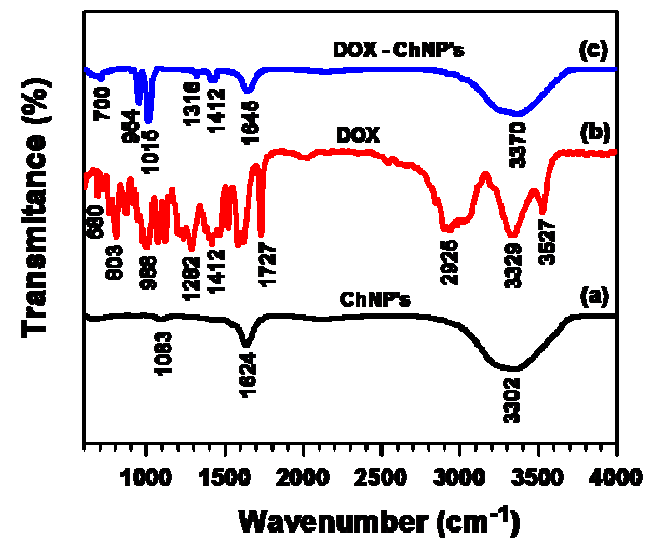

Fig. 2: FTIR spectra of a) ChNP's b) DOX c) DOX-ChNP's

Encapsulation efficiency and in vitro drug release

The DOX (DOX:Ch-1:10) encapsulated in all formulations of TPP: Ch. Encapsulation efficiencies shown in table 1. TPP:Ch (0.1:0.5) showed higher encapsulation efficiency (69 $\pm 3.24 \%)$. In vitro release of DOX were performed in phosphate buffer saline at $\mathrm{pH}-7.4$ physiological $\mathrm{pH}$ of body fluids. The average cumulative release of DOX from nanoparticles at the prefixed time interval is shown in fig. 2. The release profile was biphasic. It was apparent that in vitro DOX release showed initial burst release and slower release of DOX in the second phase. After, $48 \mathrm{~h}$ it was shown $86.6 \pm 4.78 \%$ of DOX released from ChNP's.

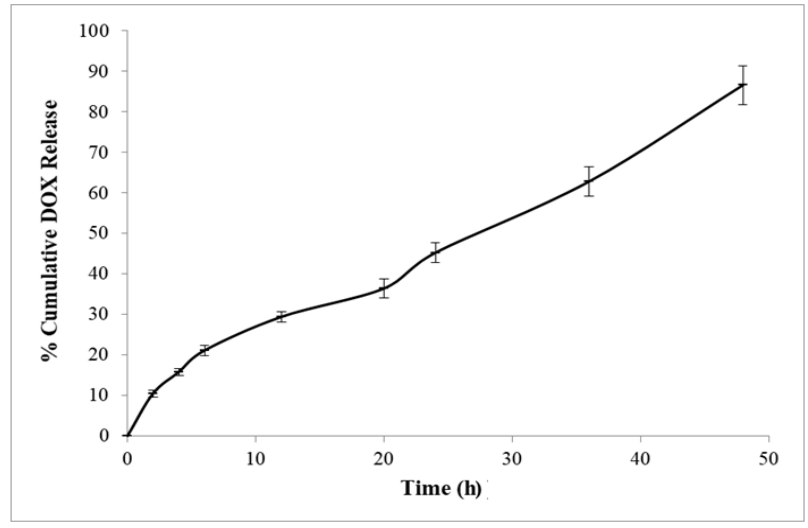

Fig. 3: In vitro release profile of DOX

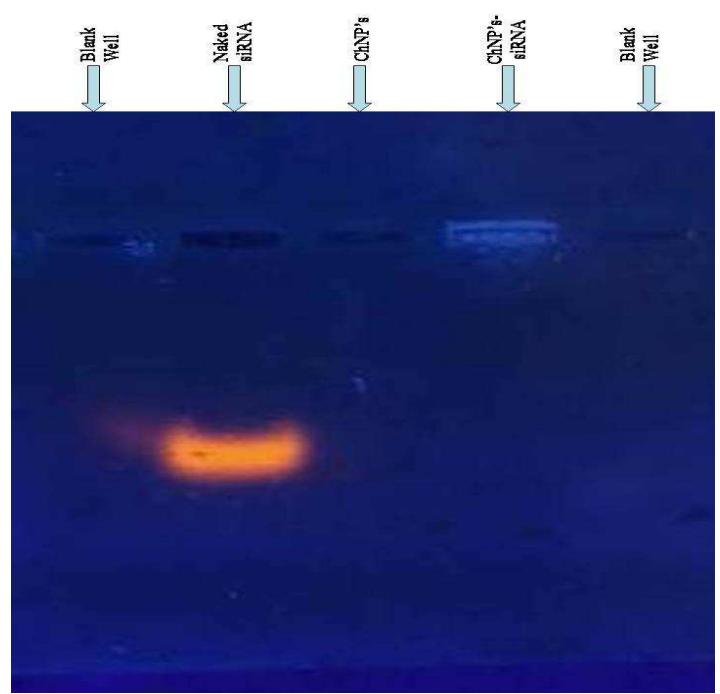

Fig. 4: Agarose gel retardation assay 


\section{Agarose gel retardation assay}

The agarose gel retardation assay was used to confirm the loading of siRNA into ChNP's. The Naked siRNA and prepared formulations such as ChNP's and siRNA-ChNP's were loaded on agarose gel as shown in fig. 4 . The results showed that naked siRNA moved with their electrophoretic mobility and shown as dark band on agarose gel. The ChNP's did not showed any migration and fluorescence. Whereas, siRNA-ChNP's showed retarded movement. The results clearly revealed that the siRNA was successfully loaded in ChNP's.

\section{CONCLUSION}

The ChNP's, DOX-ChNP's, DOX-siRNA-ChNP's were successfully prepared by ionic gelation method. The TPP:Ch ratio 1:5 was found optimum for ChNP's formulation on the basis of optimum particle size

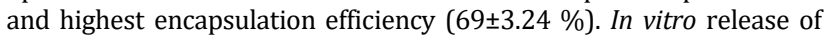
DOX were showed initial burst release and prolong sustained-release i.e. $86.6 \pm 4.78 \%$ at the end of $48 \mathrm{~h}$. The loading of siRNA in ChNP's was confirmed by agarose gel electrophoresis retardation assay. The developed DOX-siRNA-ChNP's having wide scope for further research in vitro and in vivo to treat various types of cancer such as colorectal cancer, breast cancer and other solid tumors.

\section{FUNDING}

The authors would like to acknowledge the D.Y. Patil Education Society, Institution deemed to be University, Kolhapur, for providing financial support to Dr. Gulbake (Instramural Project Grant, DYPES DU/2017/2653).

\section{AUTHORS CONTRIBUTIONS}

All the authors have contributed equally.

\section{CONFLICT OF INTERESTS}

Declared none

\section{REFERENCES}

1. Chandran SP, Natarajan SB, Chandrasekaran S, Mohd Shahimi MSB. Nano drug delivery strategy of 5-fluorouracil for the treatment of colorectal cancer. J Cancer Res Practice 2017;4:45-8.

2. Cisterna BA, Kamaly N, Choi WI, Tavakkoli A, Farokhzad OC, Vilos C. Targeted nanoparticles for colorectal cancer. Nanomedicine (Lond) 2016;11:2443-56.

3. Kim HJ, Kim A, Miyata K, Kataoka K. Recent progress in the development of siRNA delivery vehicles for cancer therapy. Adv Drug Delivery Rev 2016;104:61-77.

4. Janes KA, Fresneau MP, Marazuela A, Fabra A, Alonso MaJ. Chitosan nanoparticles as delivery systems for doxorubicin. J Controlled Release 2001;73:255-67.

5. Nogueira Librelotto DR, Scheeren LE, Vinardell MP, Mitjans M, Rolim CMB. Chitosan-tripolyphosphate nanoparticles functionalized with a $\mathrm{pH}$-responsive amphiphile improved the in vitro antineoplastic effects of doxorubicin. Colloids Surf B 2016;147:326-35.

6. Souto GD, Farhane Z, Casey A, Efeoglu E, McIntyre J, Byrne HJ. Evaluation of cytotoxicity profile and intracellular localization of doxorubicin-loaded chitosan nanoparticles. Anal Bioanal Chem 2016;408:5443-55.

7. Hellmers F, Ferguson P, Koropatnick J, Krull R, Margaritis A. Characterization and in vitro cytotoxicity of doxorubicin-loaded $\gamma$-polyglutamic acid-chitosan composite nanoparticles. Biochem Eng J 2013;75:72-8.

8. Butt AM, Amin MC, Katas H, Abdul Murad NA, Jamal R, Kesharwani P. Doxorubicin and siRNA codelivery via chitosancoated $\mathrm{pH}$-responsive mixed micellar polyplexes for enhanced cancer therapy in multidrug-resistant tumors. Mol Pharm 2016;13:4179-90.

9. Conde J, de la Fuente JM, Baptista PV. Nanomaterials for reversion of multidrug resistance in cancer: new hope for an old idea? Front Pharmacol 2013;4:134.

10. Sadreddini S, Safaralizadeh R, Baradaran B, Aghebati Maleki L, Hosseinpour Feizi MA, Shanehbandi D, et al. Chitosan nanoparticles as a dual drug/siRNA delivery system for treatment of colorectal cancer. Immunol Lett 2017;181:79-86.

11. Bumcrot D, Manoharan M, Koteliansky V, Sah DW. RNAi therapeutics: a potential new class of pharmaceutical drugs. Nature Chem Biol 2006;2:711-9.

12. Ku SH, Jo SD, Lee YK, Kim K, Kim SH. Chemical and structural modifications of RNAi therapeutics. Adv Drug Delivery Rev 2016;104:16-28.

13. Lam JK, Chow MY, Zhang Y, Leung SW. siRNA Versus miRNA as therapeutics for gene silencing. Mol Ther Nucleic Acids 2015;4:e252.

14. Saraswathy M, Gong S. Recent developments in the co-delivery of siRNA and small molecule anticancer drugs for cancer treatment. Materials Today 2014;17:298-306.

15. Ibrahim H, Farid O, Samir A, Mosaad R. Preparation of chitosan antioxidant nanoparticles as drug delivery system for enhancing of anti-cancer drug. Key Eng Materials 2018;759:92-7.

16. Calvo P, Remunan Lopez C, Vila Jato JL, Alonso MJ. Novel hydrophilic chitosan-polyethylene oxide nanoparticles as protein carriers. J Appl Polymer Sci 1997;63:125-32.

17. Sun $\mathrm{Q}$, Wang $\mathrm{X}$, Cui C, Li J, Wang Y. Doxorubicin and anti-VEGF siRNA co-delivery via nano-graphene oxide for enhanced cancer therapy in vitro and in vivo. Int $\mathrm{J}$ Nanomed 2018;13:3713-28.

18. $\mathrm{Xu} \mathrm{Y,} \mathrm{Du} \mathrm{Y.} \mathrm{Effect} \mathrm{of} \mathrm{the} \mathrm{molecular} \mathrm{structure} \mathrm{of} \mathrm{chitosan} \mathrm{on}$ protein delivery properties of chitosan nanoparticles. Int J Pharm 2003;250:215-26. 